Check for updates

Cite this: RSC Adv., 2017, 7, 25483

\title{
Enhanced light scattering effect of wrinkled transparent conductive ITO thin film
}

Received 6th March 2017 Accepted 3rd May 2017

DOI: $10.1039 / \mathrm{c} 7 \mathrm{ra02726e}$

rsc.li/rsc-advances

\author{
Chuang Wang, $\dagger^{\text {ad }}$ Haoran Zhang, (D) $\dagger^{\text {ad }}$ Fengyou Yang, ${ }^{\text {ad }}$ Yongtao Fan ${ }^{c}$ \\ and Qian Liu*abd
}

Indium tin oxide (ITO) plays an important role due to its high transparency and high conductivity in various optoelectronic thin-film devices. However, ITO has the drawback of mechanical fragility, limiting its development. Wrinkling has been considered a powerful tool for improving the flexibility and surface properties of thin films, but wrinkled ITO has not been reported so far. In this work, we fabricate uniform wrinkles on ITO and systematically study the properties of the wrinkled ITO in optics, electrics and mechanics. The wrinkled ITO shows a high optical transmittance and also a high haze value due to a remarkable wrinkle-induced enhancement of light scattering, which is beneficial for solar cells and lightemitting diodes. Our experimental results also indicate that wrinkles can effectively improve the mechanical bending performance of ITO without compromising the crystallization and electrical conductivity.

\section{Introduction}

Wrinkling, a ubiquitous natural phenomenon, occurs spontaneously when a multilayer thin-film system is under compressive stress. Previously, wrinkles have always been regarded as having a negative effect on thin-film devices. Once they appear on the surface, it usually means that some damage has been caused. But in recent years, some studies have found that wrinkle structures are also helpful for improving the surface or interface properties of thin-film devices. ${ }^{1-8}$ For example, wrinkles can enhance the luminous efficiency of organic light emitting diodes (OLEDs) ${ }^{3}$ and increase the energy harvesting efficiency of solar cells. ${ }^{4}$ Moreover, in stretchable optoelectronic devices, wrinkles can release stress and increase integral flexibility. ${ }^{2}$ Besides, wrinkled structures also play an important role in many other fields such as microfluidics, ${ }^{8}$ mechanical metrology, ${ }^{9}$ strain-engineering ${ }^{10,11}$ and so on.

In various optoelectronic devices such as OLEDs and solar cells, materials with a remarkable combination of high electrical conductivity and optical transparency are indispensable. ${ }^{12}$ Numerous materials have been exploited for transparent conductive electrodes (TCEs), including: (1) doped metal oxide films ${ }^{13-15}$ such as indium tin oxide (ITO), fluorine-doped tin oxide (FTO), aluminum-doped zinc oxide (AZO) and indium-doped zinc oxide

${ }^{a}$ CAS Center for Excellence in Nanoscience, National Center for Nanoscience and Technology, Beijing 100190, P. R. China.E-mail: liuq@nanoctr.cn

${ }^{b}$ The MOE Key Laboratory of Weak-Light Nonlinear Photonics, TEDA Applied Physics Institute and School of Physics, Nankai University, Tianjin 300457, P. R. China

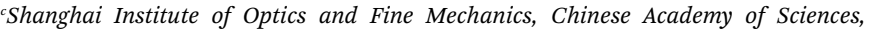
Shanghai 201800, P. R. China

${ }^{d}$ University of Chinese Academy of Sciences, Beijing 100049, P. R. China

$\dagger$ These authors contributed equally to the work.
(IZO); (2) transparent conductive polymer materials ${ }^{16}$ for example poly(3,4-ethylenedioxythiophene):poly(styrenesulfonate) (PEDOT:PSS); (3) network-type thin films of noble metal (gold or silver) nanowires ${ }^{17-19}$ or carbon nanotubes; ${ }^{20}(4)$ graphene $^{21}$ and other two-dimensional materials. Among them, ITO is the most preferred material of the past several decades because of its mature technique and excellent performance. ${ }^{12-14}$ However, the one critical drawback of ITO is that it easily cracks at very low bending strain, which limits its applications in flexible devices. Introducing wrinkle structures might be an effective strategy to improve the flexibility of ITO films and enhance other surface properties as well.

To the best of our knowledge, there is no report on studying the introduction of wrinkles into ITO for enhancing its surface performance. In this work, we fabricate random wrinkles on ITO films in a simple and low-cost way and comprehensively study the influence of the wrinkles on ITO. The results indicate that the wrinkled ITO has a huge increase in light scattering and in bending resistance, while the optical transparency, crystal structure and electrical conductivity remain unaffected.

\section{Experimental}

\section{Fabrication of wrinkled and flat ITO films}

To fabricate high quality ITO films, various methods have been proposed, such as thermal evaporation, chemical vapor deposition (CVD), pulsed laser deposition (PLD) and magnetron sputtering. Among them, magnetron sputtering is the most used method because of its good uniformity and high efficiency. In this study, we deposited ITO films by a magnetron sputtering deposition system (Kurt J. Lesker, PVD75) equipped with a loadlock. 


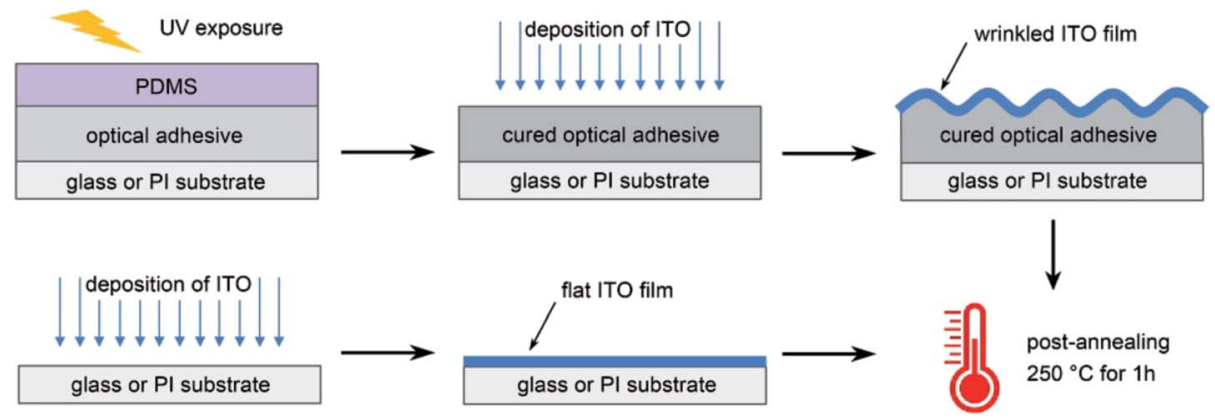

Fig. 1 The fabrication process for the wrinkled and flat ITO film.

The fabrication process for wrinkles in an ITO/adhesive bilayer on a glass or polyimide (PI) substrate is depicted in Fig. 1. Firstly, a few drops of Norland Optical Adhesive (NOA 61) were dripped onto a glass or PI substrate and covered with a flat PDMS stamp to form a flat and uniform optical adhesive film (about $20 \mu \mathrm{m}$ thick). After being exposed to ultraviolet light for $10 \mathrm{~min}$ ( $365 \mathrm{~nm}, 200 \mathrm{~mW} \mathrm{~cm}^{-2}$ ), the PDMS stamp was removed, leaving a cured optical adhesive film which was ready for the deposition of ITO. The target was an $\mathrm{In}_{2} \mathrm{O}_{3}-\mathrm{SnO}_{2}(90: 10 \mathrm{wt} \%)$ ceramic bonded to a copper backing plate. The base pressure of the vacuum system before deposition was lower than $1 \times 10^{-6}$ Torr to ensure the film quality. Then the ITO sputtering was performed in a pure argon $(100 \% \mathrm{Ar})$ atmosphere at a pressure of 5 mTorr with a DC power of $60 \mathrm{~W}$. The sputtering time was $1 \mathrm{~h}$ for depositing an ITO layer about $70 \mathrm{~nm}$ thick. During the deposition, the collision of target molecules with the NOA 61 layer can cause a local heating effect and impose isotropic compressive stress on the adhesive layer, resulting in the generation of random wrinkles on the ITO/NOA 61 bilayer. For reference, a flat ITO film was also deposited directly on a glass or PI substrate in the absence of NOA 61 according to the same sputtering process parameters. For increasing the electrical conductivity and light transmittance of the amorphous ITO films deposited, crystallization is needed by annealing the ITO at high temperature. Therefore, after deposition, both the wrinkled ITO/NOA 61 bilayer and flat ITO film were postannealed in the same vacuum chamber at a temperature of $250{ }^{\circ} \mathrm{C}$ for $1 \mathrm{~h}$ for crystallization.

Our fabrication method for wrinkles has the advantages of being a simple process and low cost, compared to methods that involve lithography or chemical synthesis for creating textured or grating structures. ${ }^{22}$

\section{Characterizations}

The surface morphologies were characterized by a laser scanning confocal microscope (LSCM, Olympus, LEXT-OLS4000) and a scanning electron microscope (SEM, Hitachi, S4800). The optical transmittance spectra of the films were recorded by a UV/VIS/NIR spectrophotometer (Varian, Cary 5000) at wavelengths from 400 to $800 \mathrm{~nm}$. The total transmittance was measured by an integrating sphere to collect the transmitted light at all angles. The specular transmittance was measured by the photo-detector only to collect the light flux along the incident light axis. The thickness of the NOA 61 layer and the deposited ITO film were measured by a surface profiler (Veeco, Dektak 150). X-ray diffraction (XRD) was performed by an X-ray diffractometer (Bruker, D8 Focus) to determine the crystal structure and phase of ITO. The square resistance of the films was measured by a Keithley System SourceMeter (2602A) attached to a manual operation probe station (TiTan Power, ZFT-160-MT).

\section{Results and discussion}

\section{Surface morphology}

Fig. 2(a) shows the flat ITO film (left) and the wrinkled ITO/NOA 61 bilayer (right) on a glass substrate (size: $24 \times 24 \times 0.15 \mathrm{~mm}$ ) after annealing. Obviously, both samples are transparent in

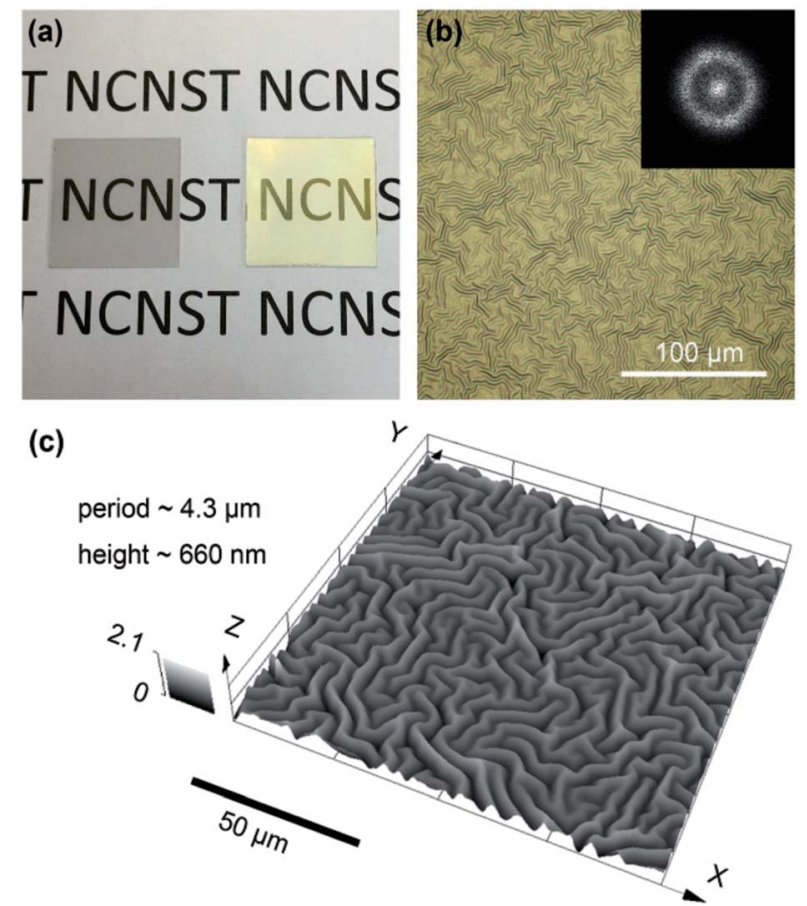

Fig. 2 (a) Digital photo of flat ITO film (left) and wrinkled ITO/NOA 61 bilayer (right) on the same glass substrate. (b) Optical microscope image of the wrinkled ITO surface, and (inset) corresponding FFT analysis showing its periodicity. (c) Magnified three-dimensional morphology of ITO wrinkles measured by LSCM. 
visible light. From the optical microscope image and the corresponding Fast Fourier Transform (FFT) analysis in Fig. 2(b), we can observe that the ITO wrinkles exhibit random orientation and a uniform distribution. The wrinkles are periodical with an intrinsic wavelength, as shown in the inset of Fig. 2(b), which is determined by the modulus and thickness of the two layers, while the amplitude of the wrinkles depends on the bilayer system and the strain value. ${ }^{1}$ Here the wrinkle period is about $4.3 \mu \mathrm{m}$ and the peak-to-valley height is about $660 \mathrm{~nm}$, according to the threedimensional morphologic characterization shown in Fig. 2(c). The periodicity and randomness of the ITO wrinkles are beneficial for both light extraction and harvesting. In addition, our wrinkled structure has a microscale period and a submicron height, offering a moderate aspect ratio of about 0.15 , which is very suitable for the use of TCEs in optoelectronic devices. In principle, a too small aspect ratio will not benefit the surface performance enhancement, while a too big aspect ratio may also degrade the device efficiency because of the underfill and air gaps in the above spin-coated active layer. ${ }^{22}$

\section{Enhanced effect of light scattering}

Optical properties are a key performance indicator of ITO films and crucially depend on the surface morphology. The flat ITO on a glass substrate is highly transparent and barely scatters the incident light, resulting in a specular transmission. The wrinkled ITO on a glass substrate is also transparent, but scatters a large part of the incident light, which leads to a diffuse transmission. In other words, all the light transmitted through the flat ITO is propagated along the incident direction, while most of the light that passes through the wrinkled ITO is deflected from the propagation path. Fig. 3(a) describes this distinction. The total transmittance of the flat ITO and the wrinkled ITO/NOA 61 measured with an integrating sphere is shown in Fig. 3(b). According to the data, the wrinkled sample even has a slightly higher optical transmittance (about 90\%) than the flat one. This wrinkle-induced enhancement of optical transmission may be caused by the light-trapping at the valleys of the wrinkles. Besides the total transmittance, haze is also frequently used to quantify the percentage of scattered light in the total transmitted light. The haze is calculated as: ${ }^{23}$

$$
\text { Haze }=\frac{T_{\text {sca }}}{T_{\text {tot }}} \times 100 \%=\frac{T_{\text {tot }}-T_{\text {spe }}}{T_{\text {tot }}} \times 100 \%
$$

where $T_{\text {tot }}, T_{\text {sca }}$ and $T_{\text {spe }}$ are the total transmittance, the scattering transmittance and the specular transmittance, respectively. The haze values of the two samples are plotted in Fig. 3(c), which indicates that the flat ITO has a very low optical haze value, while the wrinkled sample has a high haze value up to $60 \%$. This implies that our wrinkled ITO/NOA 61 film has ultrahigh optical transparency and simultaneously an ultrahigh optical haze. Considering that ITO has a relatively wide absorption range, ${ }^{\mathbf{1 3}}$ our wrinkled ITO film should have a similar high transmittance and high haze in a broader spectral range. Since the wrinkles are isotropic and evenly distributed, the resultant enhancement in transparency and haze is homogeneous. It is critical to combine the haze and transmittance for different applications. In particular, this excellent dual performance in transmittance and haze is most suitable for solar cells. ${ }^{4,22,23}$ On the one hand, the high transparency ensures that nearly all the sunlight can enter the photovoltaic active layer. On the other hand, the light scattering can increase the light absorption path in the active layer, which can remarkably improve the conversion efficiency of the devices. In the area of LEDs, such a feature is likewise helpful to extract confined light and remove the spectral dependency on the viewing angle, ${ }^{3}$ which is of critical significance in achieving high energy efficiency.

\section{Improvement of bending performance}

In addition to providing a huge increase in light scattering, wrinkles can also relieve mechanical stress and thus increase the robustness of devices. ${ }^{2,4}$ ITO is a kind of ceramic material and naturally has poor bending performance. Here we fabricated the flat ITO film and wrinkled ITO/NOA 61 bilayer on flexible PI substrates (size: $24 \times 24 \times 0.15 \mathrm{~mm}$ ), and investigated their tolerance to mechanical bending. Bend tests for the flat and wrinkled ITO samples were performed with a prespecified bending strain $(\varepsilon)$, which is defined as: ${ }^{4}$

$$
\varepsilon=\frac{t}{2 r} \times 100 \%
$$

where $t$ denotes the sample thickness, and $r$ is the bending radius. Fig. 4(a) and (c) show that there are no cracks on the surface of both the flat sample and the wrinkled sample before mechanical bending $(\varepsilon=0)$. Fig. 4(b) shows that cracks occur densely normal to the bend direction on the sample surface (a)

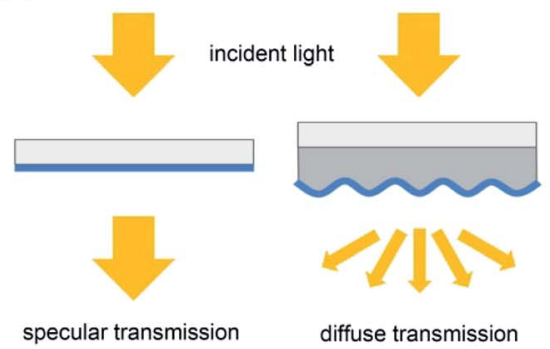

(b)

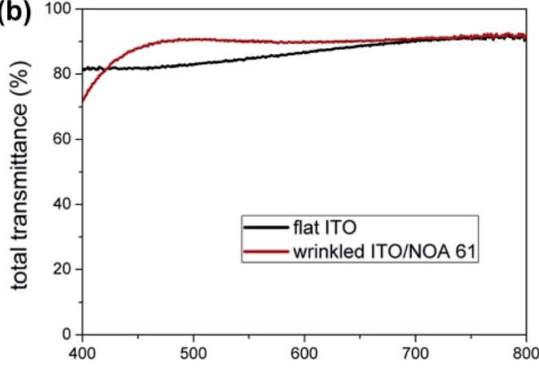

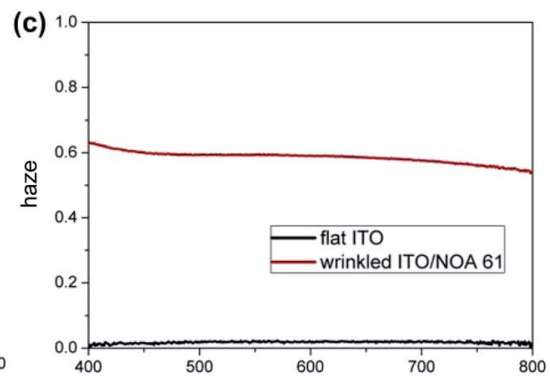

Fig. 3 (a) Schematic diagram showing specular transmission of flat ITO and diffuse transmission of wrinkled ITO/NOA 61. Total transmittance (b) and haze (c) of flat ITO and wrinkled ITO/NOA 61. 

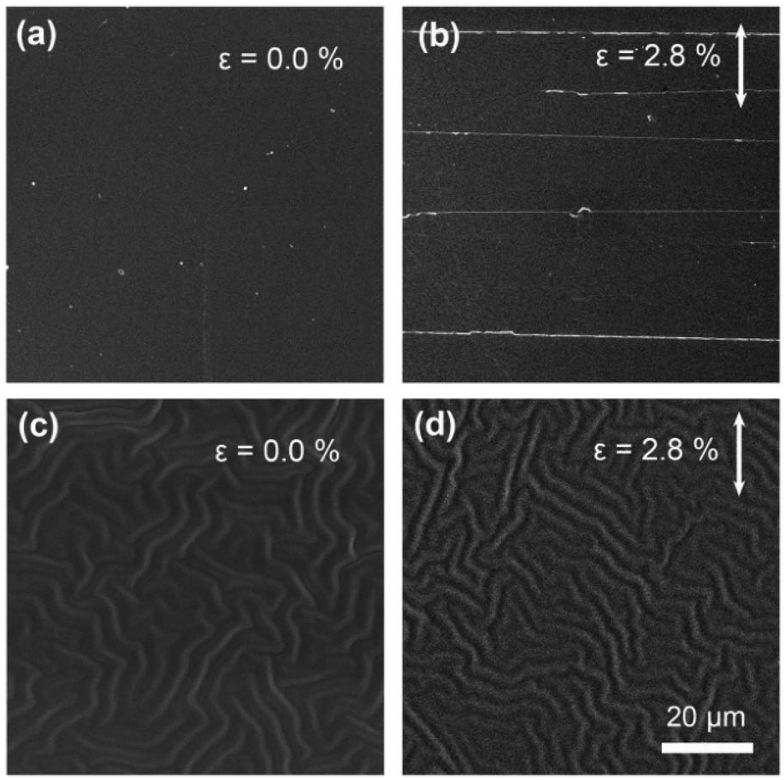

Fig. 4 Scanning electron microscope images of the ITO surface of the flat ( $a$ and $b$ ) and wrinkled ( $c$ and d) sample before ( $a$ and $c$ ) and after (b and d) the bend tests at a bending strain of $\sim 2.8 \%$. The arrow indicates the deformation direction.

after bending 10 times at $\varepsilon=2.8 \%$ along the arrow direction, indicating the mechanical fragility of the flat ITO film. In contrast, no obvious cracks were observed on the surface of the wrinkled sample in Fig. 4(d), indicating that these wrinkles can effectively alleviate strain and prevent fractures or cracks from forming. This enhancement of mechanical properties is extremely favourable for the flexibility and the long-term performance of ITO devices. Moreover, owing to the isotropic orientation of the wrinkled structure, such mechanical enhancement is thus available in all directions, which is particularly applicable to various wearable devices.

\section{Crystal structure and electrical conductivity}

Whether the wrinkling process will affect the crystal structure and electrical properties of ITO is also an unavoidable issue that needs attention. The XRD spectra of our flat and wrinkled ITO films after being annealed at $250{ }^{\circ} \mathrm{C}$ for $1 \mathrm{~h}$ under vacuum are shown in Fig. 5(a). The cubic structure with diffraction peaks corresponding to the (llll $\left.\begin{array}{lll}2 & 1\end{array}\right),\left(\begin{array}{lll}2 & 2 & 2\end{array}\right),\left(\begin{array}{lll}4 & 0 & 0\end{array}\right),\left(\begin{array}{lll}4 & 4 & 0\end{array}\right)$ and (lll $\left.\begin{array}{ll}6 & 2\end{array}\right)$ planes reflects that both annealed films have been crystallized. ${ }^{13,14}$ The intensity of some characteristic peaks is not very strong because the ITO thickness is relatively thin and the annealing temperature is moderate. From the XRD structural investigation, there is no evident difference between the flat and wrinkled ITO films in the crystalline structure, which indicates that the wrinkling process doesn't affect the ITO crystallization during annealing.

The degree of crystallization can significantly affect the properties of ITO, especially the electrical conductivity. When the film is amorphous, a large number of electrons are bound within a small range by the non-uniform structure and the defects. Once the ITO becomes crystalline, these bound electrons are released, consequently increasing the carrier concentration and the mobility of the ITO film. In our study, the flat and the wrinkled ITO films exhibit nearly equivalent electrical conductivity, as shown in Fig. 5(b), due to undergoing the same crystallization. It should be noted that the square resistance of the wrinkled ITO film is a little higher than that of the flat film, which may be attributed to the wrinkles slightly extending the surface area of the ITO film, but this subtle distinction won't impact the actual use.

\section{Conclusions}

In summary, we have investigated the wrinkled transparent conductive ITO thin film, which is naturally formed when ITO is deposited on a layer of NOA 61. Experimental results indicate that the wrinkled ITO film exhibits not only ultrahigh transparency but also an ultrahigh haze value, which result from the wrinkle-enhanced light scattering enhancement effect. It should be specially pointed out that the light scattering enhancement effect will benefit the light harvesting efficiency of solar cells as well as the light extracting efficiency of LEDs. In addition, the wrinkles can also increase the flexibility of the ITO film and thus can improve the device stability and tolerance. Moreover, the wrinkled ITO films still maintain an unchanged light transparency, electrical resistance and crystal structure. These results indicate that the wrinkled ITO will have great potential applications in the fields of solar cells, LEDs and flexible devices. We believe that the work can also provide
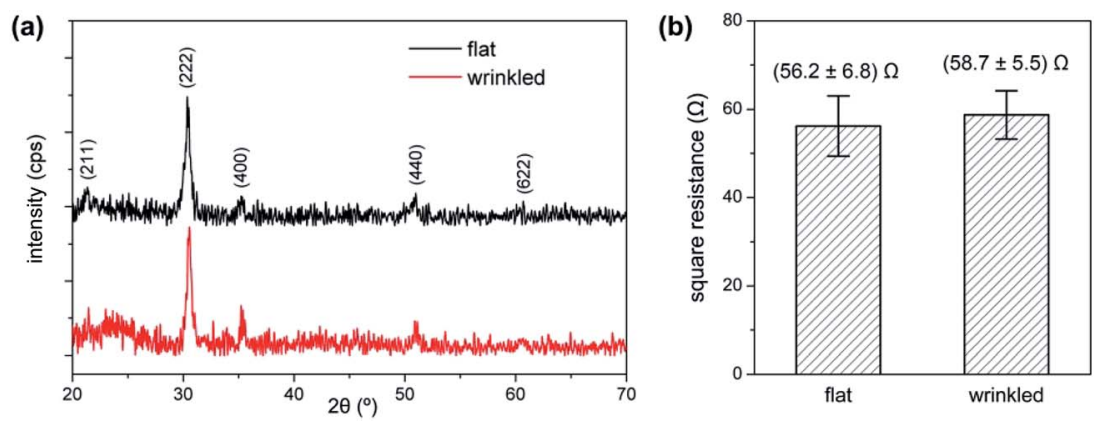

Fig. 5 X-ray diffraction spectra (a) and square resistance (b) of flat and wrinkled ITO. Each set of data in (b) shows the average value and standard deviation of five identical samples. 
helpful guidance for increasing the performances of other transparent conductive films.

\section{Acknowledgements}

This project is supported by the National Key Research Program of China (2016YFA0200403), the CAS Strategy Pilot Program (XDA 09020300), and the National Natural Science Foundation of China (10974037, 61405223).

\section{Notes and references}

1 S. Yang, K. Khare and P.-C. Lin, Adv. Funct. Mater., 2010, 20, 2550-2564.

2 Y. Sun, W. M. Choi, H. Jiang, Y. Y. Huang and J. A. Rogers, Nat. Nanotechnol., 2006, 1, 201-207.

3 J. Moon, E. Kim, S. K. Park, K. Lee, J.-W. Shin, D.-H. Cho, J. Lee, C. W. Joo, N. S. Cho, J.-H. Han, B.-G. Yu, S. Yoo and J.-I. Lee, Org. Electron., 2015, 26, 273-278.

4 J. B. Kim, P. Kim, N. C. Pégard, S. J. Oh, C. R. Kagan, J. W. Fleischer, H. A. Stone and Y.-L. Loo, Nat. Photonics, 2012, 6, 327-332.

5 C. F. Guo, V. Nayyar, Z. Zhang, Y. Chen, J. Miao, R. Huang and Q. Liu, Adv. Mater., 2012, 24, 3010-3014.

6 A. R. Shugurov, A. I. Kozelskaya and A. V. Panin, $R S C A d v$, 2014, 4, 7389.

7 J. Yin and C. Lu, Soft Matter, 2012, 8, 6528.

8 K. Khare, J. Zhou and S. Yang, Langmuir, 2009, 25, 1279412799.

9 J. Y. Chung, A. J. Nolte and C. M. Stafford, Adv. Mater., 2011, 23, 349-368.

10 H. Zhang, F. Yang, J. Dong, L. Du, C. Wang, J. Zhang, C. F. Guo and Q. Liu, Nat. Commun., 2016, 7, 13743.
11 S. Yang, C. Wang, H. Sahin, H. Chen, Y. Li, S. S. Li, A. Suslu, F. M. Peeters, Q. Liu, J. Li and S. Tongay, Nano Lett., 2015, 15, 1660-1666.

12 A. Kumar and C. Zhou, ACS Nano, 2010, 4, 11-14.

13 H. Kim, C. M. Gilmore, A. Piqué, J. S. Horwitz, H. Mattoussi, H. Murata, Z. H. Kafafi and D. B. Chrisey, J. Appl. Phys., 1999, 86, 6451-6461.

14 C. C. Wu, C. I. Wu, J. C. Sturm and A. Kahn, Appl. Phys. Lett., 1997, 70, 1348-1350.

15 D. Liu, S. Ren, X. Ma, C. Liu, L. Wu, W. Li, J. Zhang and L. Feng, RSC Adv., 2017, 7, 8295-8302.

16 Y. H. Kim, C. Sachse, M. L. Machala, C. May, L. MüllerMeskamp and K. Leo, Adv. Funct. Mater., 2011, 21, 10761081.

17 C. F. Guo, Q. Liu, G. Wang, Y. Wang, Z. Shi, Z. Suo, C.-W. Chu and Z. Ren, Proc. Natl. Acad. Sci. U. S. A., 2015, 112, 1233212337.

18 C. F. Guo, T. Sun, Q. Liu, Z. Suo and Z. Ren, Nat. Commun., 2014, 5, 3121.

19 Y. Song, Y. Jiang, L. Shi, S. Cao, X. Feng, M. Miao and J. Fang, Nanoscale, 2015, 7, 13694-13701.

20 Z. Wu, Z. Chen, X. Du, J. M. Logan, J. Sippel, M. Nikolou, K. Kamaras, J. R. Reynolds, D. B. Tanner and A. F. Hebard, Science, 2004, 305, 1273-1276.

21 X. Wang, L. Zhi and K. Müllen, Nano Lett., 2008, 8, 323-327. 22 K. S. Nalwa, J. M. Park, K. M. Ho and S. Chaudhary, Adv. Mater., 2011, 23, 112-116.

23 Z. Fang, H. Zhu, Y. Yuan, D. Ha, S. Zhu, C. Preston, Q. Chen, Y. Li, X. Han, S. Lee, G. Chen, T. Li, J. Munday, J. Huang and L. Hu, Nano Lett., 2014, 14, 765-773. 